\title{
Hematopoietic Progenitor Cells from Cord Blood
}

National Cancer Institute

\section{Source}

National Cancer Institute. Hematopoietic Progenitor Cells from Cord Blood. NCI

Thesaurus. Code C133329.

Blood collected from the umbilical cord as a source of hematopoietic progenitor cells. 